\title{
Using Horizontal and Slant Lidar Measurements to Obtain Calibrated Aerosol Scattering Coefficients from a Coastal Lidar in Hawaii*
}

\author{
J. N. Porter, B. Lienert, And Shiv K. Sharma \\ School of Ocean and Earth Science and Technology, Hawaii Institute of Geophysics and Planetology, \\ University of Hawaii at Manoa, Honolulu, Hawaii
}

(Manuscript received 6 August 1999, in final form 25 February 2000)

\section{ABSTRACT}

\begin{abstract}
Sea salt aerosol concentrations in the clean marine boundary layer can be considered spatially homogeneous when averaged over space and time. Using this assumption, horizontal and slant lidar measurements are carried out at a Hawaii coastal site allowing accurate retrieval of the marine aerosol scattering coefficient. It is found that when the lidar calibration or the aerosol phase function is adjusted so that the derived scattering coefficients are constant with range, then the derived aerosol scattering coefficients are correct and agree with nephelometer measurements (within 25\%) and sun photometer (within 7\%). Examples of this calibration process are given.
\end{abstract}

\section{Introduction}

Although lidars are ideal for mapping the spatial distribution of aerosol concentrations, efforts to obtain quantitative estimates of the aerosol extinction or scattering coefficients are not straightforward. The major difficulties arise from the uncertainty in the aerosol backscatter-to-extinction ratio and the lidar calibration. The aerosol backscatter-to-extinction ratio depends on the aerosol phase function at $180^{\circ}$ (backward scattering angle) and the aerosol single-scatter albedo, which determines the aerosol absorption versus scattering (Lenoble 1993). Uncertainty in the lidar calibration (laser intensity and receiver efficiency) is another source of uncertainty and can change with time depending on the state of the optics (clean or dirty).

Following Reagan (1995) the lidar equation can be written as

$$
\begin{aligned}
& n(r)=\left(\frac{\lambda \delta_{\lambda}}{h c} A_{r} \Delta r E_{o}\right) \frac{\beta(r) T(r)^{2}}{r^{2}} \text { or } \\
& n(r)=C \frac{\beta(r) T(r)^{2}}{r^{2}},
\end{aligned}
$$

where $n(r)$ is the photoelectrons received from distance

* School of Ocean and Earth Science and Technology Contribution Number 5258.

Corresponding author address: Dr. John N. Porter, School of Ocean and Earth Science and Technology, University of Hawaii at Manoa, 2525 Correa Road, Honolulu, HI 96822.

E-mail: jporter@soest.hawaii.edu $r$ and range bin $\Delta r$. Here, $E_{\mathrm{o}}$ is the laser pulse energy at wavelength $\lambda$. The detector quantum efficiency is $\delta_{\lambda}$, $h$ is Planck's constant, $c$ is the speed of light, $A$ is the telescope effective area, $\beta(r)$ is the back scattering coefficient at range $r$, and $C$ is the lidar calibration coefficient. Then $T(r)^{2}$ is the atmospheric transmission term and is given by

$$
T(r)^{2}=e^{-2 \int \sigma_{e}(r) d r} .
$$

The extinction coefficient $\left[\sigma_{e}(r)\right]$ is the sum of the scattering and absorption coefficients $\left[\sigma_{\mathrm{s}}(r)+\sigma_{\mathrm{a}}(r)\right]$. The scattering coefficient, $\sigma_{\mathrm{s}}(r)$, is the sum of the aerosol and molecular scattering coefficients $\left[\sigma_{\mathrm{as}}(r)+\sigma_{\mathrm{ma}}(r)\right]$. The absorption coefficient is also due to aerosol absorption and molecular absorption $\left[\sigma_{\mathrm{as}}(r)+\sigma_{\mathrm{ma}}(r)\right]$. The molecular absorption coefficient $\left[\sigma_{\mathrm{ma}}(r)\right]$ is negligible as lidar wavelengths are typically chosen where little molecular absorption occurs. For coastal regions at clean remote sites, sea spray is the dominant cause of extinction. Under these conditions, the aerosol absorption coefficient, $\sigma_{\text {aa }}(r)$, is negligible and the aerosol scattering and extinction coefficients are effectively identical.

The lidar return signal decreases primarily with the inverse of the distance squared but also due to the transmission squared term. In Eq. (1) the backscattering coefficient is given by

$$
\beta(r)=\frac{P_{m}(r) \sigma_{\mathrm{ms}}(r)+P_{a}(r) \sigma_{\mathrm{as}}(r)}{4 \pi}
$$

where

$P_{m}(r)=$ the molecular phase function at distance $r$, $P_{a}(r)=$ the aerosol phase function at distance $r$, 
$\begin{aligned} \sigma_{\mathrm{ms}}(r)= & \text { the molecular scattering coefficient at dis- } \\ \text { tance } r & \end{aligned}$ $\sigma_{\text {as }}(r)=$ the aerosol scattering coefficient at distance $r$.

As the aerosol properties and the lidar calibration are often not known, numerous techniques have been proposed to convert lidar backscatter measurements into aerosol extinction. As summarized by Reagan et al. (1989), these include the slope method (Collis 1966), modeled transmission method (Russell et al. 1979), slant path method (Sandford 1967), and various methods using a relationship between extinction and backscatter (Hitschfeld and Bordan 1954; Fernald et al. 1972; Klett 1981). The last approach has been popular because it creates a relationship between the aerosol extinction coefficient $\sigma_{\text {as }}(r)$ and the aerosol backscattering coefficient $\beta_{a}(r)$, thus reducing the number of independent variables. Klett (1981) assumed a power law relationship between the aerosol extinction and the aerosol backscattering coefficient given by

$$
\beta(r)=C_{2} \sigma_{\mathrm{as}}(r)^{k},
$$

where the constants $C_{2}$ and $k$ depend on the aerosol type. Estimates of the $k$ constant ranged from 0.67 to 1 (Klett 1981). Klett also proposed using a known value of the aerosol scattering at a distant point and solving for the along-path extinction with a backward stepping approach, which he showed to be more stable than the forward stepping approach. Ferguson and Stephens (1983) extended this method performing iterative calculations in order to minimize the difference in the measured range corrected signal, $S_{m}=\ln \left[r^{2} n(r)\right]$, and the range corrected signal $\left(S_{c}\right)$ calculated from the derived aerosol scattering coefficients. This approach was found to be very sensitive to the lidar calibration (Hughes et al. 1985) and the $C_{2}$ parameters used in the power law relation (4), which describes $\beta_{r}$ and $\sigma_{r}$.

If the aerosols are horizontally homogeneous then horizontal or slant measurements can be used to provide boundary values to constrain the aerosol extinction coefficients (Zhang and $\mathrm{Hu}$ 1997) and even to estimate the aerosol phase function (Young et al. 1993). In this paper, modeling efforts and measurements will be shown for horizontal measurement in marine air. It will be shown that for a horizontally homogeneous atmosphere, the aerosol scattering coefficient can, in theory, be derived with an accuracy of up to $5 \%$ depending on the range to which the calibration is extended and the accuracy to which the lidar derived scattering coefficients can made constant with range.

\section{Forward stepping algorithm}

In order to derive the aerosol scattering coefficient from our coastal lidar measurements we have implemented a simple forward stepping algorithm with a modified horizontal shot technique. As sea salt is the dominant source of extinction at our Hawaii coastal site, we assume the aerosol absorption is negligible and the aerosol scattering and extinction are equal (aerosol single-scatter albedo of 1). The forward stepping lidar inversion begins by assuming a fixed aerosol transmission, $T_{a}(r)$, from the lidar out to range, $r \sim 300 \mathrm{~m}$. This is needed because in the near field region the scattered light cannot be properly focused on the detector. The error introduced by uncertainty in this initial transmission is small and can be removed by using an iterative approach; however, this was not done for these studies as the effect was found to be negligible. The molecular transmission, $T_{m}(r)$, from the lidar out to the initial range $(300 \mathrm{~m})$ is calculated [Eq. (2)] based on pressure and temperature. Beyond this near-field region the inversion is begun by calculating the aerosol scattering coefficient in the next sample bin at distance $r(r-\Delta r / 2$ to $r+$ $\Delta r / 2)$ using

$\sigma_{\mathrm{as}}(r)=\left\{n(r) \frac{\left(4 \pi r^{2}\right)}{C\left[T_{m}(r-\Delta r) T_{a}(r-\Delta r)\right]^{2}}-P_{m} \sigma_{\mathrm{ms}}\right\} \frac{1}{P_{a}}$,

where Eqs. (1)-(3) have been combined and solved for the aerosol scattering coefficient. The aerosol transmission from the lidar through the bin is $T_{a}(r)=T_{a}(r-$ $\Delta r) \Delta T_{a}(r)$ where $\Delta T_{a}(r)=e^{-\sigma_{\text {as }}(r) \Delta r}$ using the aerosol extinction calculated in Eq. (5). Similarly a new molecular transmission, is calculated by $T_{m}(r)=T_{m}(r-$ $\Delta r) \Delta T_{m}(r)$ where $\Delta T_{m}(r)=e^{-\sigma_{\mathrm{ms}}(r) \Delta r}$. The molecular scattering coefficient is computed at the proper pressure and temperature following Lenoble (1993) using the hypsometric equation for the height dependence. Next a new aerosol scattering coefficient is calculated in the next sample bin using Eq. (5) again. The process is repeated out to the end of the lidar measurement. This forward stepping approach has no restriction on the decreasing transmission and can accumulate large error at longer distances. The error comes from using an incorrect aerosol phase function (at $180^{\circ}$ ) or wrong lidar calibration (Hughes et al. 1985). Here it is assumed that the lidar is properly aligned and the laser beam is within the collection cone of the receiver optics.

\section{Modeling tests}

In order to test this forward stepping approach, we generate pseudo-lidar data by using Eqs. (1)-(3). The aerosol phase function (phase function at $180^{\circ}$ ) value was set to 0.65 , to represent marine sea salt. The aerosol is assumed to have no absorption so that the scattering and extinction coefficient are the same (aerosol singlescatter albedo $=1$ ). The aerosol is assumed to be horizontally homogeneous with a scattering coefficient of $5 \times 10^{-5} \mathrm{~m}^{-1}$. The lidar calibration $C$ is set to $5 \times 10^{-3}$ $\mathrm{m}^{3}$, which is appropriate for our lidar system in the A/D converter configuration (discussed below). The molecular scattering coefficient was set to $1.211 \times 10^{-5} \mathrm{~m}^{-1}$ corresponding to surface pressure $(1013 \mathrm{mb})$ and $532-$ 


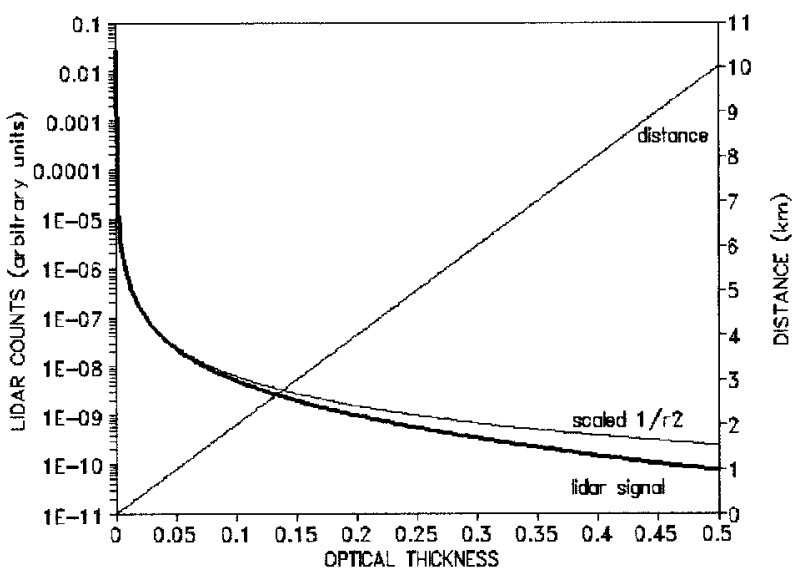

FIG. 1. Simulated lidar counts as a function of optical thickness for a horizontal lidar measurement. The aerosol scattering coefficien used in the calculations is constant with range with a value of $5 \times$ $10^{-5} \mathrm{~m}^{-1}$. Also shown is a scaled $1 / r^{-2}$ curve for comparison. The straight line and the right side give the distance assuming an aerosol scattering coefficient of $0.5 \times 10^{-5} \mathrm{~m}^{-1}$.

nm wavelength. Further discussion on the appropriateness of these aerosol and lidar values is given below. Figure 1 shows the modeled lidar return signal from a horizontal lidar measurement with homogeneous aerosol. The lidar signal has been plotted versus the total optical thickness and the distance is shown on the right side. Plotting the data as a function of optical thickness allows for different aerosol loading. The relationship between the lidar signal and the $1 / r^{2}$ term (scaled to fit on the same plot) is also shown as a function of optical thickness. It can be seen that the lidar signal closely follows a $1 / r^{2}$ drop off out to an optical depth of 0.1 but at further optical distances the atmospheric transmission becomes significant. This simple relationship between the lidar signal and a $1 / r^{2}$ dropoff is routinely used in our system for coarse alignment of our optics.

Noise in lidar measurements is either electrical or from coarse digitization. For measurements in the marine boundary layer the signal is large and the digitization noise dominates. The A/D system we use has 12 bit digitization (0-4096 counts) and the digitization noise is half of a count. In order to simulate the digitization noise, positive and negative oscillating values were added to the calculated lidar signal in Fig. 1. The magnitude of the noise was set to 0.5/4096 times the lidar counts at $300 \mathrm{~m}$ where the lidar signal first becomes useful due to near field telescope effects. It is assumed that the gain is adjusted so that maximum counts occur at the 300-m distance.

Using the forward stepping approach described above, the aerosol scattering coefficient is derived from the model-generated data using correct and incorrect values of the aerosol phase function (at $180^{\circ}$ ). These results are shown in Fig. 2 where it can be seen that the error increases with distance as wrong values of the phase function are used. The noise introduced now ap-

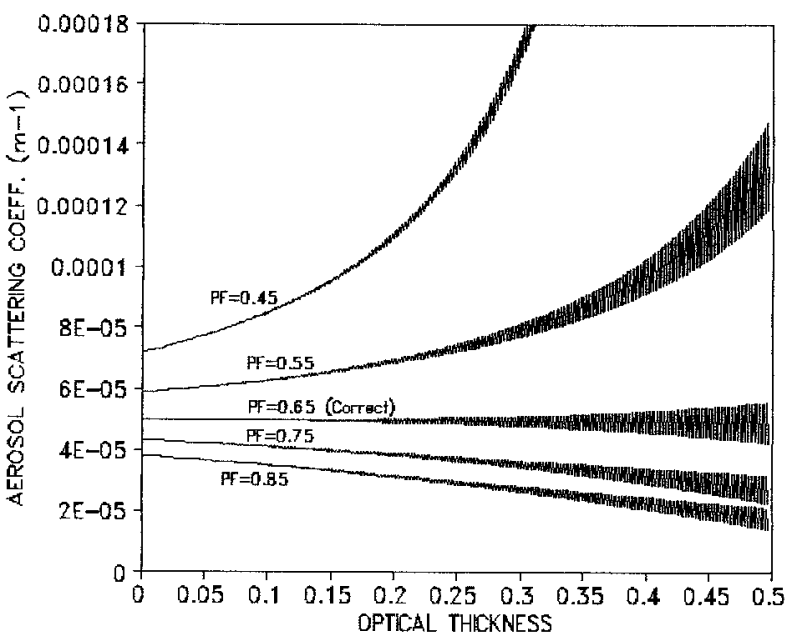

FIG. 2. Aerosol scattering coefficient derived from the data shown in Fig. 1 using the forward stepping algorithm. The range is plotted as aerosol optical thickness. Different aerosol phase function values were used (see label) in the inversion which caused the derived values to stray from the correct value $\left(5 \times 10^{-5} \mathrm{~m}^{-1}\right)$. A 5 -point smoothing has also been applied to reduce the noise that was introduced in the model data.

pears much larger as a result of the $r^{2}$ term in Eq. (5). As expected, using the correct aerosol phase function value, yields the correct aerosol scattering coefficient (5 $\left.\times 10^{-5} \mathrm{~m}^{-1}\right)$ at all distances. Greater error results if the aerosol phase function is underestimated than if overestimated. If the aerosol phase function is overestimated the dropoff with range is not so strong and might appear as a straight line if measurements are not made to large enough optical depths. These results are similar to those presented in Hughes et al. (1985) where they studied the dependence of the derived extinction coefficient with the constants used in the power law relationship given in Eq. (4).

The digitization noise introduced into the lidar signal has a big effect in the derived aerosol scattering coefficients, which increases with distance as shown in Fig. 2. In Fig. 2 5-point smoothing of the aerosol scattering coefficients has been applied. Larger smoothing values can be used if desired and they do not affect the final results other than to reduce the digitization noise. Here we have smoothed derived aerosol scattering coefficients rather than the original lidar counts.

Using incorrect values for the lidar calibration value (C) will cause similar errors. Figure 3 shows larger errors in the derived aerosol scattering coefficients when the lidar calibration value is too small and these errors become larger with range. As $C$ becomes too large the error is not as dramatic and does not change as much with distance. The derived aerosol scattering coefficients at all distances are correct $\left(5 \times 10^{-5} \mathrm{~m}^{-1}\right)$ when the derived aerosol scattering coefficients is constant with range and incorrect when it is not constant. This effect was shown by Hughes et al. (1985) but did not appear in the sensitivity studies performed by Klett 


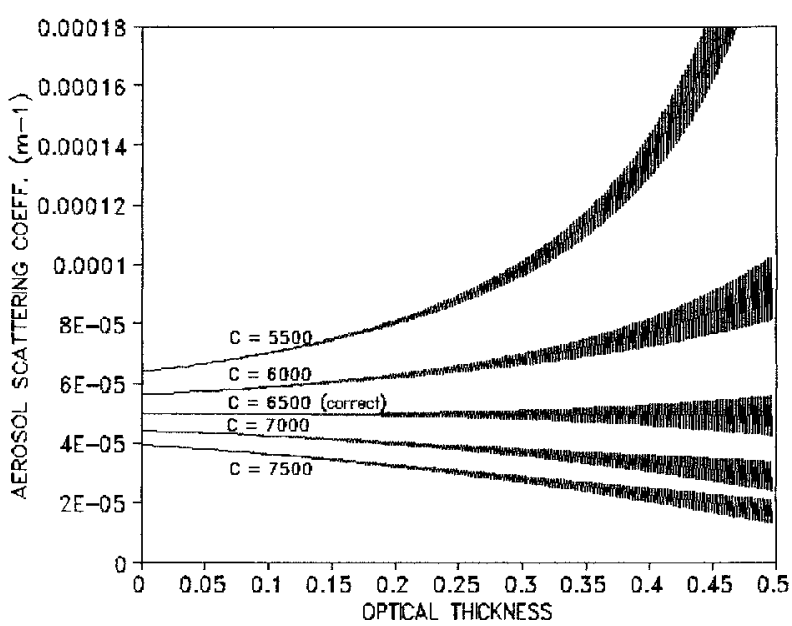

FIG. 3. Aerosol scattering coefficient derived as a function of optical thickness assuming the correct lidar calibration and several incorrect lidar calibrations. The correct value is $5 \times 10^{-5} \mathrm{~m}^{-1}$. A 5-point smoothing has been applied to the derived aerosol scattering coefficients shown here to reduce the noise.

(1981, their Fig. 1). It is also important to note that the correct aerosol scattering coefficient and a constant response with range can be obtained using incorrect values for both the lidar calibration and the aerosol phase function values since the errors can cancel. For this reason, it is necessary to make a best guess at either the aerosol phase function or the lidar calibration and adjust the other until the derived aerosol scattering coefficients have a constant response with range. This method provides a simple "manual" iterative approach to obtain accurate aerosol scattering coefficient values for a relatively homogeneous air mass. This method is less prone to errors than the slope method (Collis 1966), which can have large fluctuations in the derived aerosol scattering coefficients.

\section{Coastal lidar measurements in Hawaii}

The forward-stepping horizontal calibration technique described above is now applied to actual lidar measurements. The system consist of a coaxial lidar employing a 30-cm Schmidt-Cassegrain telescope and a $20-\mathrm{Hz} \mathrm{Nd}$ :YAG laser $\left(80 \mathrm{~mJ}\right.$ pulse $\left.{ }^{-1}\right)$ at $532 \mathrm{~nm}$. The beam diameter is expanded to a diameter of $4 \mathrm{~cm}$ and directed by two motorized scanning mirrors for azimuth and zenith control. The energy of each lidar pulse is normalized by measuring a fraction of the pulse energy with a fast diode. The signal from the diode is digitized at a high rate and stored under computer control. Further details of system and software are given in Lienert et al. (1999).

The aerosol near Hawaii can be summarized as "clean marine" most of the year with the exception of springtime Asian dust-pollution episodes and occasional Hawaii volcano plume events (during southeast winds). The aerosol in the clean marine trade wind atmosphere has typical accumulation mode concentrations of $0.5 \mu \mathrm{g}$ $\mathrm{m}^{-3}$ and coarse mode sea salt concentrations of 5-10 $\mu \mathrm{g} \mathrm{m}^{-3}$ (Pruppacher and Klett 1998). Based on Mie calculations from aerosol models (Porter and Clarke 1997), the sea salt aerosol contributes $\sim 70 \%$ of the optical depth, while the accumulation mode contributes $\sim 30 \%$ for typical trade wind conditions $\left(\sim 7 \mathrm{~m} \mathrm{~s}^{-1}\right.$ wind speeds). The sea salt aerosol has very little absorption so that the aerosol scattering and extinction coefficients are essentially the same. In the vertical, the largest salt concentrations occur in the lowest $30 \mathrm{~m}$ (Blanchard et al. 1984; Daniels 1989). From $30 \mathrm{~m}$ up to cloud base $(\sim 500 \mathrm{~m})$ the sea salt aerosol concentrations are fairly constant (Woodcock 1953). Above cloud base the aerosol concentrations decrease slightly and remain roughly constant up to the trade wind inversion. Above the inversion the aerosol scattering coefficients are quite low and little or no sea salt is present with the exception of springtime when Asian dust and pollution is carried over Hawaii. Aerosol optical depths at the Mauna Loa observatory (3.3-km altitude) during the most of the year are typically 0.015 . If we assume this aerosol is spread out over $15-\mathrm{km}$ altitude then the average aerosol scattering coefficients in the free troposphere are $1 \times 10^{-6}$ $\mathrm{m}^{-1}$. This is consistent with nephelometer measurements at the Mauna Loa observatory, which have clean values of $1 \times 10^{-6} \mathrm{~m}^{-1}$ or smaller for summer, fall, and winter periods (Hoffmann et al. 1995).

Lidar measurements were made at two coastal sites on the windward side of Oahu (Makai pier and Bellows Beach). At these coastal sites breaking waves create heavy spray which rapidly coats the lidar optics. Under strong trade winds, we have found the lidar calibration can change significantly in less than 30 min owing to accumulation of salt spray on the scanning mirrors. This rapid coating of our mirrors means we cannot assume a fixed lidar calibration value. For this reason we assume a constant aerosol phase function value and modify the lidar calibration to obtain accurate aerosol scattering coefficient values.

In order to calculate the value of the aerosol phase function we used Mie theory and sea salt aerosol models of Porter and Clarke (1997). The sea salt aerosol hygroscopic growth and index of refraction corresponding to $75 \%$ relative humidity (Shettle and Fenn 1979). This aerosol model is based on the salt size distribution measurements taken by Woodcock (1953) for the range from 1 to $50 \mu \mathrm{m}$ and optical particle counter measurements from 0.2- to 1.0- $\mu \mathrm{m}$ diameter (Porter and Clarke 1997). The aerosol model employs a combination of two lognormal curves to best fit the sea salt observations at different wind speeds. The model is representative of sea salt in the lower $500 \mathrm{~m}$ of the boundary layer. Using the aerosol models for different wind speeds, the aerosol phase function value (at $180^{\circ}$ ) is calculated and shown in Fig. 4 for different aerosol scattering coefficient values (wind speeds). The 12 points correspond to the 12 Beaufort wind scales or wind speeds of $0.4-1.5,1.6-$ 


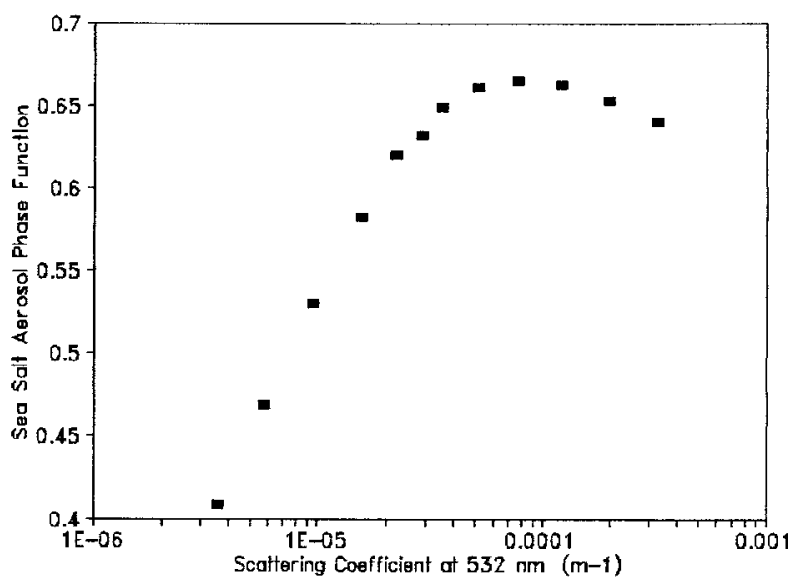

FIG. 4. Aerosol phase function values $\left(180^{\circ}\right.$ scattering angle) for different sea salt aerosol concentrations going from wind Beaufort scale 1-12. Mie theory was used and the corresponding aerosol scattering coefficients are shown on the $x$ axis. All Mie calculations were done with aerosol at equilibrium at $75 \%$ relative humidity.

$3.3,3.4-5.4,5.5-7.9,8-10.7,10.8-13.8,13.9-17.1$, $17.2-20.7,20.8-24.4,24.5-28.4,28.5-33.5,>33.5 \mathrm{~m}$ $\mathrm{s}^{-1}$ (Woodcock 1953). The variations of the phase function value seen in Fig. 4 is caused by the salt size distribution shifting to larger sizes at higher wind speeds. Nephelometer measurements at our site typically have aerosol scattering coefficient values between $3 \times 10^{-5}$ to $6 \times 10^{-4} \mathrm{~m}^{-1}$ for normal trade wind conditions. Typical trade wind speeds are also approximately $7 \mathrm{~m} \mathrm{~s}^{-1}$. Based on these factors, an aerosol phase function value of 0.65 was chosen as being representative of sea salt.

Figure 5a shows lidar data collected on January 1997, in which the lidar was pointed out to sea in the approximate upwind direction. Measurements were integrated for $3 \mathrm{~min}$ using a photon counter. The measurements have been plotted as the logarithm of the range corrected signal, $S_{m}=\ln \left[r^{2} n(r)\right]$. While a straight line can be fit to the data, large fluctuations exist, which raise questions about how well these measurements can be used to obtain a lidar calibration. Next the forward stepping inversion (described above) was applied to the dataset and the aerosol scattering coefficient was derived as a function of distance (Fig. 5b). The lidar calibration was first adjusted so that the aerosol scattering coefficients at greater optical thickness (greater distance) were too large $\left(C=3.7 \times 10^{-3} \mathrm{~m}^{3}\right)$. Then the lidar calibration was lowered until the derived aerosol scattering coefficients were constant with range (best estimate at $C=$ $\left.4.9 \times 10^{-3} \mathrm{~m}^{3}\right)$. As an example of an overestimate, the lidar calibration was also set too large $\left(C=6 \times 10^{-3}\right.$ $\mathrm{m}^{3}$ ). Based on the constant values with range, the best estimate of the aerosol scattering coefficient is $1 \times 10^{-4}$ $\mathrm{m}^{3}$. During the lidar measurements, simultaneous aerosol scattering measurements were made with a Radiance Research nephelometer instrument, which measured an average aerosol scattering coefficient of $7.5 \times 10^{-5} \mathrm{~m}^{-1}$ at $500 \mathrm{~nm}$. The position of the nephelometer was such
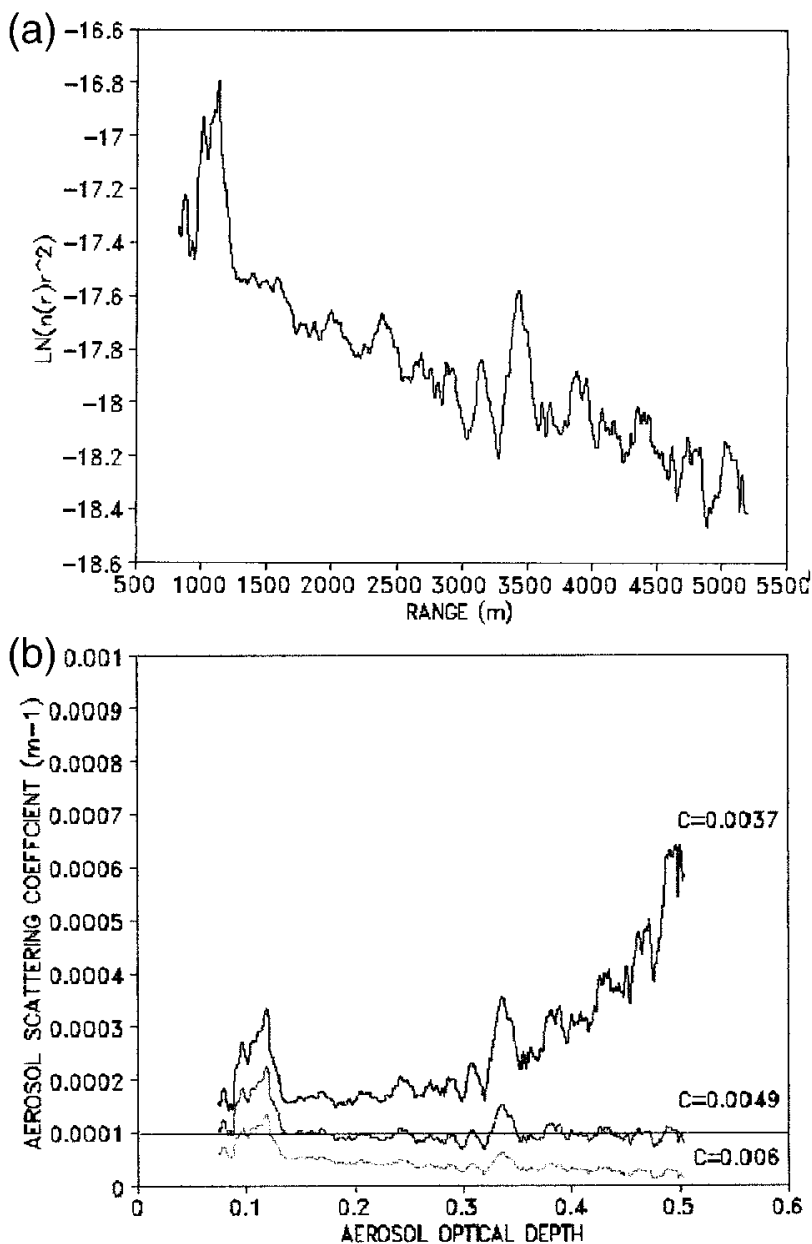

FIG. 5. (a) Three-minute average lidar measurements collected on the windward side of Oahu pointing the lidar horizontally out over the ocean. The $x$ axis shows the range and the $y$ axis is the logarithm of the photon counter counts corrected by $\left(r^{2}\right)$. It is obvious that large fluctuations are present but that they vary about a background value. (b) Aerosol scattering coefficient plotted as a function of aerosol optical depth for lidar measurements made Makai pier, Oahu. Different lidar calibrations (C) were used in the forward stepping algorithm. Nephelometer measurements at the lidar site had values of $7.5 \times 10^{-5} \mathrm{~m}^{-1}$

that it measured the same air, which was present over the open ocean and was not affected by the spray from an isolated reef, which caused the increase in scatter, seen at optical depth 0.1 in Fig. 5. The nephelometer measurement is $\sim 25 \%$ lower than the best estimate from the lidar measurement. The lower value for the nephelometer is expected due to gravitational settling and impaction losses on bends (Porter et al. 1992). In this study we operated the nephelometer facing into the wind without any inlet tubing. Several tests were performed and we noticed $\sim 20 \%$ signal decrease when a 5 -in. inlet was used. The nephelometer also underestimates the scatter because the limited range of scattering angles $\left(7^{\circ}-170^{\circ}\right)$ missing some of the scattered light (Anderson et al. 1996). 
Both Figs. 3 and 5 show that an overestimate of the lidar calibration causes the derived scattering values to be too low but these values do not change significantly with distance. Because of this weak distance dependence, it is difficult to distinguish between the correct and incorrect lidar calibration if the lidar calibration is too large. On the other hand, an underestimate of the lidar calibration has a large effect with distance and the error is obvious. Therefore, it is best to start with a lidar calibration value, which is too small, and gradually increase it until the derived scattering coefficient is constant with range.

Another example of this forward-stepping horizontal calibration technique is shown in Figs. 6a and 6b using data collected with the lidar in a scanning mode on 26 July 1999. For these scanning lidar measurements the system had a weaker laser, the averaging for each range measurement was short ( $1 \mathrm{~s}$ vs $3 \mathrm{~min}$ in Fig. 5), the sampling bins were larger, and the counts per photon were lower (using A/D converter vs photon counter). These differences caused the lidar calibration to be different from the measurements shown in Figs. 5 and 6. Figures 6a,b show the same scanning lidar measurements (pointed out to sea) with different lidar calibration coefficients. The aerosol phase function was set to 0.65 for both cases. The lidar calibration is incorrect in Fig. $6 \mathrm{a}$ as the derived aerosol scattering coefficients are too large at distance. In Fig. $6 \mathrm{~b}$ the derived aerosol scattering coefficients appear constant with distance so the calibration value and the aerosol scattering coefficients are believed to be correct. Up to $\sim 800 \mathrm{~m}$ height the aerosol scattering coefficient is $\sim 6 \times 10^{-5} \mathrm{~m}^{-1}$ with an optical thickness of 0.048 . From 800 to $\sim 1700 \mathrm{~m}$ height the aerosol scattering coefficient is $\sim 3 \times 10^{-5} \mathrm{~m}^{-1}$ with an optical thickness of 0.027 . Above $1700 \mathrm{~m}$ the aerosol scatter is too low to measure with the rapid scanning mode (scanning at $1^{\circ} \mathrm{s}^{-1}$ ). Aerosol optical depth measurements at the Mauna Loa Observatory typically measure 0.01 to 0.02 (at $500 \mathrm{~nm}$ ) during this time of the year. If we combine these we get a total aerosol optical depth of 0.09 for the lidar measurements at a wavelength of $532 \mathrm{~nm}$. Simultaneous sun photometer measurements at $500-\mathrm{nm}$ wavelength were taken and values ranged from 0.077 to $0.09( \pm 0.01)$. This difference between the lidar and sun photometer values is within the expected error of the lidar retrieval (further discussion below).

\section{Several layers of different aerosol}

In the discussion so far we have assumed that sea salt is the dominant scatterer in the clean marine boundary layer. In the free troposphere the aerosol changes dramatically so the aerosol phase function should change as well. In our forward-stepping approach we have not accounted for a change in the phase function along the path. Figure 7 illustrates this problem. The solid line shows the true scattering coefficient from which the modeled lidar data were generated. The modeled data for the first section had an aerosol phase function of 0.6 and 0.4 for the second section. The thin line shows the scattering coefficient retrieved using the forward-stepping approach and a constant phase function value of 0.6 throughout the whole distance. It can be seen that the derived scattering coefficient is correct for the first layer but is too small for the second layer and gets smaller with distance. If the first layer is thicker and has an optical thickness of 0.5 instead of 0.025 (Fig. 7b) then the aerosol scattering coefficient derived for the second layer is fairly accurate even though the phase function value used in the retrieval is wrong for this upper layer. Finally if the aerosol optical thickness of the first layer is made larger (Fig. 7c) then the derived aerosol scattering coefficient for the upper layer is too large. This dependence of the derived aerosol scattering coefficient on the optical distance of the first layer creates the possibility of testing the derived aerosol scattering coefficient at upper layers by looking at different slant paths (Spinhirne et al. 1980; Reagan et al. 1989). Looking vertically, the path through the lower layer is short. Looking at a slant, the path through the lower layer is larger, such as Fig. 7b or 7c. If derived aerosol scattering in the upper layer varies significantly with slant angle then the aerosol phase function for the upper layer is different from the lower layer. While the approach described above sounds feasible, in the field we are required to point the lidar at fairly large zenith angles to obtain any significant change in the optical distance through the first layer. To obtain these high zenith angles (low elevation angles) the conditions must be essentially cloud free, which is rare at our Hawaii coastal site. Therefore we have not been able to test this approach at our site. Reagan et al. (1989) point out this same effect and state that the optical depths must be greater than 0.03 for the slant path method to work.

\section{Discussion}

The methods discussed here apply to marine sea salt aerosol, which has no absorption at $532 \mathrm{~nm}$. We have also assumed that the aerosol scatter is horizontally homogeneous over the open ocean. Waves breaking near the coastline have been avoided as much as possible in this effort. The calibration approach consists in making a best guess at the aerosol phase function and lidar calibration and making forward stepping calculations of the aerosol scattering coefficient. Through a "manual" iterative approach, the lidar calibration is adjusted until the derived aerosol scattering coefficients are constant with range. The accuracy of this approach depends on the range to which measurements and analysis are extended. The further the analysis is extended, the more accurate the derived aerosol scattering coefficients will be. The accuracy also depends on the ability of the operator to adjust the lidar calibration to obtain constant aerosol scattering coefficients with range. Marine aero- 
(a)
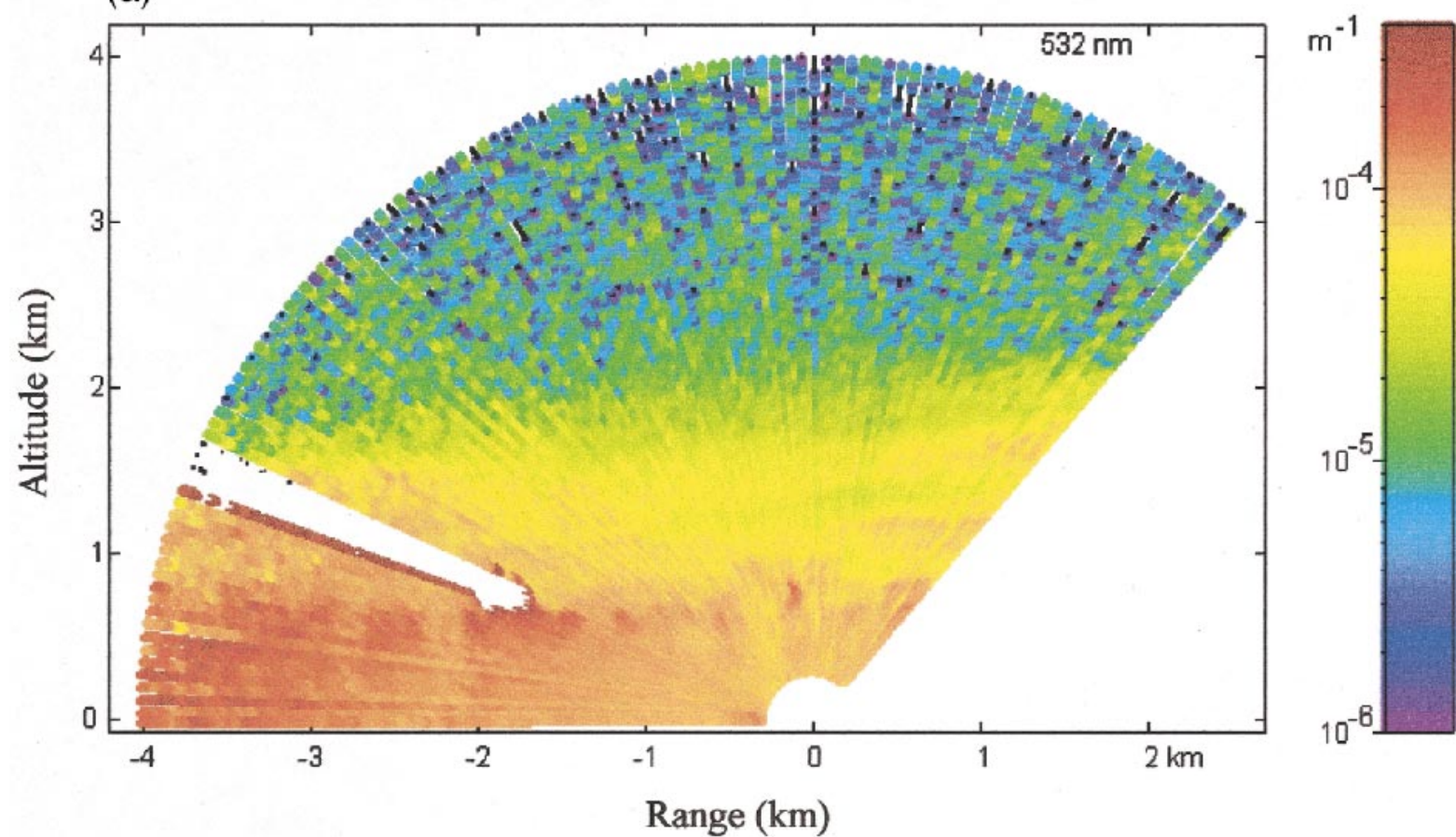

(b)
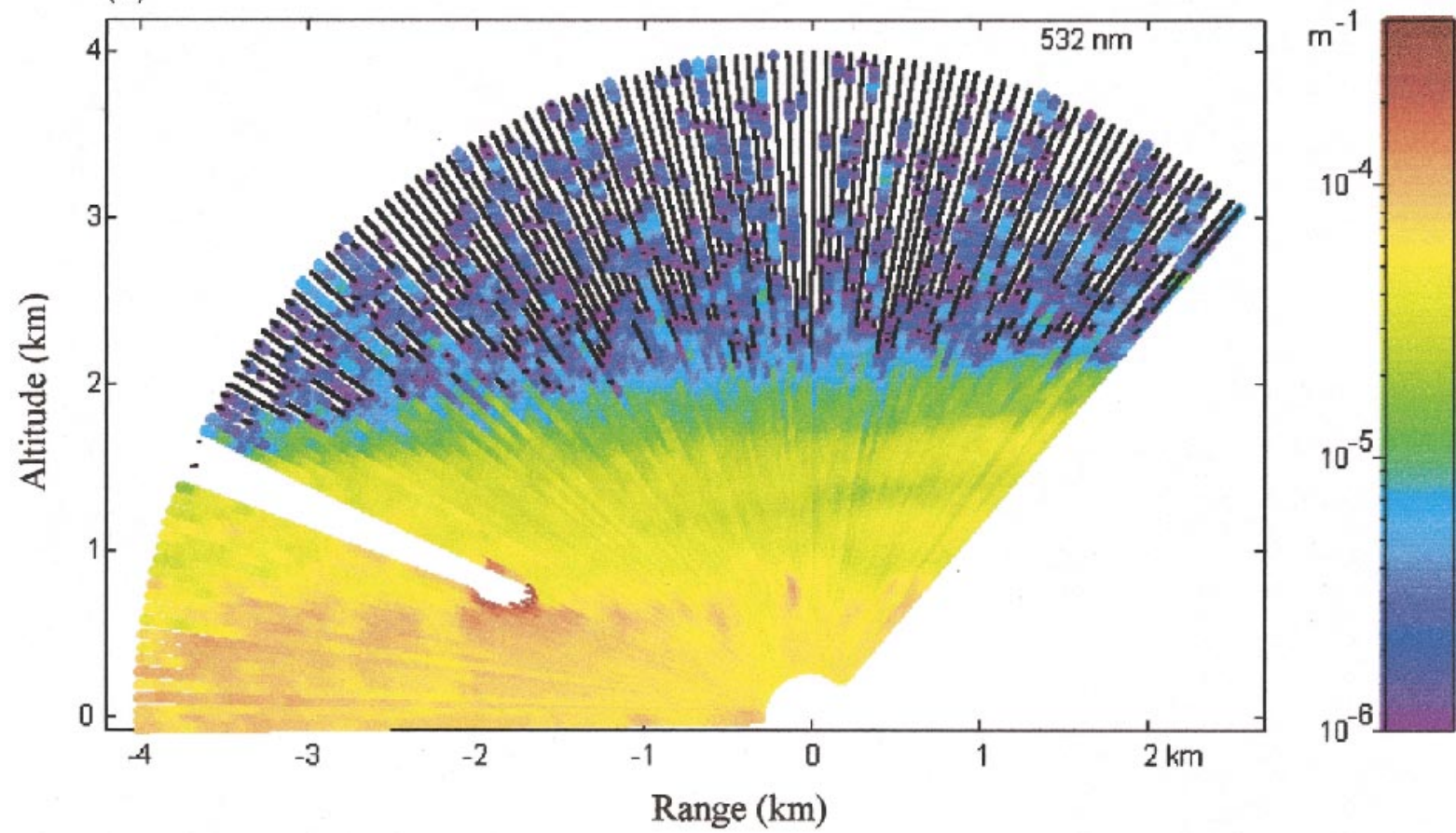

Fig. 6. (a) Aerosol scattering coefficients derived from scanning lidar measurements using the forward stepping algorithm. The $x$ axis is horizontal distance from the lidar. The $y$ axis is vertical distance from the surface. The aerosol phase function was set to 0.65 and the lidar calibration was set to 5000. Notice that the boundary layer aerosol scattering coefficients at 3-km horizontal range are larger than those at $1-\mathrm{km}$ horizontal range. This indicates the lidar calibration is too small. (b) Same as Fig. 6a but now the lidar calibration was set to 6500 . Now the derived aerosol scattering coefficients are horizontally homogeneous in the lower boundary layer (below $1 \mathrm{~km}$ ). 

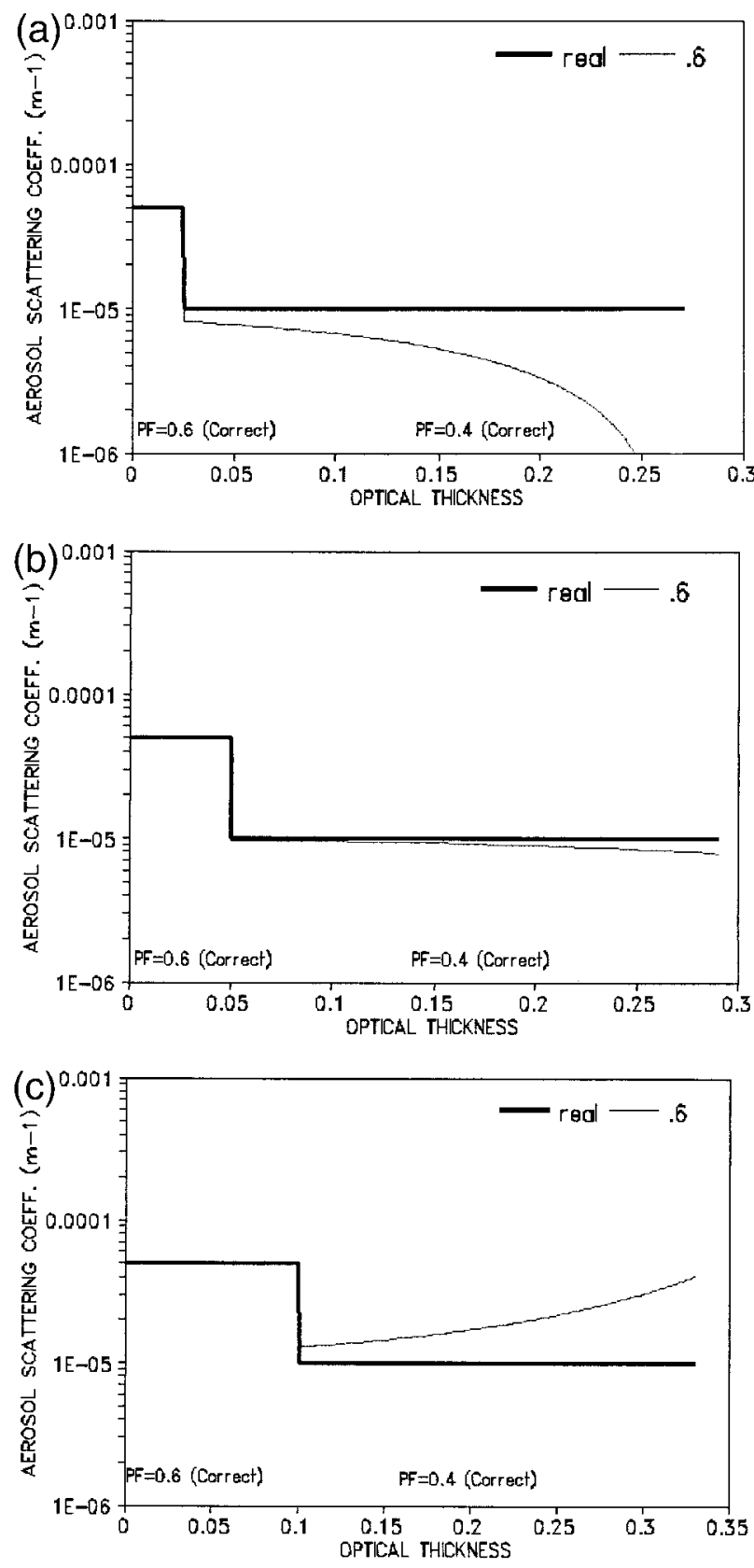

FIG. 7. (a) Thick line shows the model aerosol scattering coefficient with distance. The aerosol phase function was set to 0.6 in the first layer and 0.4 in the second layer. Thin line shows the aerosol scattering coefficient derived using the forward stepping algorithm. An aerosol phase function of 0.6 was used in the inversion for the whole distance. (b) Thick line shows the model aerosol scattering coefficient with distance. The aerosol phase function was set to 0.6 in the first layer and 0.4 in the second layer. Thin line shows the aerosol scattering coefficient derived using the forward stepping algorithm. An aerosol phase function of 0.6 was used in the inversion for the whole distance. (c) Thick line shows the model aerosol scattering coefficient with distance. The aerosol phase function was set to 0.6 in the firs layer and 0.4 in the second layer. Thin line shows the aerosol scattering coefficient derived using the forward stepping algorithm. An aerosol phase function of 0.6 was used in the inversion for the whole distance.

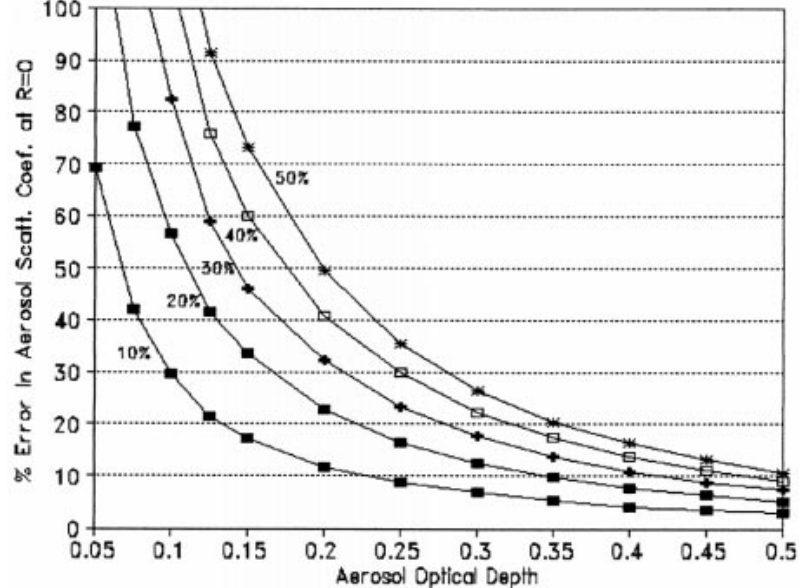

FIG. 8. Error in the derived aerosol scattering coefficient at range zero vs the aerosol optical depth (distance) to which the inversion is extended. The line labeled $10 \%$ corresponds to a condition where the derived aerosol scattering coefficients varies by $10 \%$ from range zero to the aerosol optical depth given on the $x$ axis.

sol can be considered as homogeneous when averaged but small-scale variations do exist as shown in Figs. 5 and 6 . These small-scale variations make the manual fitting less accurate. The overall accuracy of this approach is illustrated in Fig. 8, which shows the error in the derived aerosol scattering coefficients at range zero plotted versus the optical thickness to which the lidar calibration is extended. Each line corresponds to a percent change in the derived aerosol scattering coefficient from range zero to each point on the $x$ axis. As an example, in Fig. 5b it is difficult to be certain that lidar calibration has been adjusted properly (constant with range) due to small-scale fluctuations. If we assume the derived aerosol scattering coefficients vary less than $40 \%$ (from range 0 to the end of the lidar measurement), then from Fig. 8 we find the error in the retrieved aerosol scattering coefficient is less than $10 \%$ since it extended out to an optical depth of 0.5 . On the other hand, in Fig. $6 \mathrm{~b}$ the aerosol scattering coefficients from the best fit are $\sim 7 \times 10^{-5} \mathrm{~m}^{-1}$ and extend out to $\sim 4.3 \mathrm{~km}$. This corresponds to an aerosol optical depth of 0.344. Assuming the lidar calibration has been adjusted so that the derived aerosol scattering coefficients vary less than $40 \%$ (from range 0 to the end of the lidar measurement) then the derived aerosol is accurate to within $\sim 17 \%$. This illustrates the benefit of making extended range calibration measurements as well as averaging for longer duration to minimize small-scale variations in aerosol scatter.

Several complications must be avoided in carrying out this manual calibration process. It is necessary to begin with a lidar calibration value that is too small and gradually increase the value. If the manual calibration process were initiated with lidar calibration value that is large then the derived aerosol scattering coefficients 
are fairly constant with range and could be mistaken as the correct value (see Fig. 3).

The assumption that the aerosol is well mixed is only valid under certain conditions. For our calibration efforts we have pointed the lidar upwind into the trade winds from the island of Oahu. Because of the remoteness of the islands, it is assumed that the aerosol is horizontally homogeneous marine sea salt aerosol. Measurements on the downwind side of the island or other large objects are likely to have less homogeneous aerosol. Figures 5 and 6 show substantial aerosol variability can still be present in the undisturbed trade winds. These small-scale turbulent eddies are most likely caused by vertical wind shear and to a lesser extent to temperature instability (thermals). Because of these turbulent eddies, it is desirable to sample for longer duration to reduce the variability when making horizontal lidar calibration measurements.

The aerosol scattering coefficients derived from the lidar can be compared with those calculated from aerosol models (Fig. 4). The wind speed was $9.9 \mathrm{~m} \mathrm{~s}^{-1}$ and the relative humidity was $74 \%$ during the lidar measurements shown in Fig. 6. The winds were measured on the beach at the lidar site. The winds off the coastline are probably $50 \%$ greater $\left(\sim 15 \mathrm{~m} \mathrm{~s}^{-1}\right)$ than those we measured due to the blocking effect of the trees and the island mountain range. Figure 4 gives a scattering coefficient of $3.6 \times 10^{-5} \mathrm{~m}^{-1}$ for this wind speed. The lidar inversion suggests larger values closer to $6-7 \times$ $10^{-5} \mathrm{~m}^{-1}$. We do know that the lidar derived optical depth (0.09) shown in Fig. 6 overestimates the average sun photometer measurements $(0.084)$ by only $7 \%$ that is within the expected error of the lidar inversion. We do not have a certain explanation for the difference between modeled and lidar derived aerosol scattering coefficients. It is possible that the Woodcock measurements slightly underestimate the size distribution at each wind speed. During his aircraft sampling, Woodcock (1953) collected salt measurements at cloud base in regions where there were no trade wind clouds overhead. When he flew below trade wind clouds, drizzle would ruin his glass slide samples (A. H. Woodcock 1987, personal communication). Cloud drizzle is frequently seen in our lidar measurements on the windward side of Oahu. These larger size aerosol/drops would not appear in the Woodcock measurements. This increase in the scattering coefficient due to drizzle may be the reason the lidar derived values are larger than the model calculations.

In this study we have assumed the aerosol phase function value from Mie calculations and marine aerosol models. If an incorrect aerosol phase function is assumed, the correct aerosol scattering coefficients is still obtained but an incorrect value of the lidar calibration is needed to make the aerosol scattering coefficients constant with range. Ideally we would like to begin with the correct aerosol phase function value. Although we believe the salt aerosol models are realistic, we do not have actual measurements of the aerosol phase function at $180^{\circ}$ scattering angle. Doherty et al. (1999) have measured the aerosol extinction to backscatter ratio $(K)$ at Cheeka Peak Observatory, Washington. For clean marine aerosol conditions, their values of $K$ had an average of 21.1 with a standard deviation of 3.7 and no significant aerosol absorption (aerosol single scatter albedo equal to 1). For these nonabsorbing aerosol, $K=4 \pi / P_{a}$ giving an average aerosol phase function of 0.595 . We have chosen a phase function value of 0.65 , which is slightly higher than the Doherty et al. (1999) value and higher than what corresponds to the standard trade wind case in Fig. 4 for several reasons. The Doherty et al. measurements must bring the aerosol through inlets and losses for the largest sea salt aerosol occurs. The Woodcock aerosol size distribution measurements were made below cloud free areas with little drizzle. Therefore we expect the size of the aerosol-drizzle measured by the lidar to be larger than those measured by Doherty or Woodcock. Figure 4 also shows that the aerosol phase function values are fairly constant for larger sea salt conditions (higher wind speeds) so that fixed value is approximately correct for a range of wind speeds (spray conditions). For these reasons we have increased the value from 0.6 to 0.65 . Direct external measurements are needed to test this assumption.

\section{Conclusions}

If the aerosols are horizontally homogeneous, then it is possible to apply a simple forward stepping algorithm to accurately derive aerosol scattering coefficient values. The correct aerosol scattering coefficients can be obtained by adjusting either the lidar calibration or the aerosol phase function value. Either correct or incorrect values of the aerosol phase function and lidar calibration can provide accurate aerosol scattering coefficients as long as the derived values are constant with range. Comparisons between lidar derived aerosol scattering coefficients and nephelometer measurements show the lidar values are $25 \%$ higher. Comparisons with sun photometers measurements show the lidar values are $\sim 7 \%$ higher which is within the expected error of the lidar inversion.

Acknowledgments. The work carried out here was funded by an ONR Grant \# N00014-96-1-0317. We are grateful to Dr. Antony Clarke for the loan of the Radiance Research nephelometer.

\section{REFERENCES}

Anderson, T. L., and Coauthors, 1996: Performance characteristics of a high-sensitivity three-wavelength total scatter/backscatter nephelometer. J. Atmos. Oceanic Technol., 13, 967-986.

Blanchard, D. C, A. H. Woodcock, and R. J. Cipriano, 1984: The vertical distribution of the concentration of sea salt in the marine atmosphere near Hawaii. Tellus, 36B, 118-125. 
Collis, R. T. H., 1966: Lidar: A new atmospheric probe. Quart. J. Roy. Meteor. Soc., 92, 220-230.

Daniels, A., 1989: Measurements of atmospheric sea salt concentrations in Hawaii using a Tala kite. Tellus, 41B, 196-206.

Doherty, S. J., T. L. Anderson, and R. J. Charlson, 1999: Measurement of the lidar ratio for atmospheric aerosols with a $180^{\circ}$ backscatter nephelometer. Appl. Opt., 38, 1823-1832.

Ferguson, J. A., and D. H. Stephens, 1983: Algorithms for inverting lidar returns. Appl. Opt., 22, 3673-3675.

Fernald, F. G., B. M. Herman, and J. A. Reagan, 1972: Determination of aerosol height distribution by lidar. J. Appl. Meteor., 11, 482489.

Hitchfeld, W., and J. Bordan, 1954: Errors inherent in the radar measurements of rainfall at attenuating wavelengths. J. Meteor., 11, $58-67$.

Hoffmann, D. J., J. T. Peterson, and R. M. Rosson, 1995: Climate Monitoring and Diagnostics Laboratory: Summary Report 19941995. NOAA Rep. 23, 161 pp.

Hughes, H. G., J. A. Ferguson, and D. H. Stephens, 1985: Sensitivity of lidar inversion algorithm to parameters relating atmospheric backscatter and extinction. Appl. Opt., 24, 1609-1613.

Klett, J. D., 1981; Stable analytical inversion solution for processing lidar returns. Appl. Opt., 20, 211-220.

Lenoble, J., 1993: Atmospheric Radiative Transfer. Deepak, 532 pp.

Lienert, B. R., J. N. Porter, and S. K. Sharma, 1999: Real time analysis and display of scanning lidar scattering data. Mar. Geod., 22, $259-265$.

Porter, J. N., and A. D. Clarke, 1997: Aerosol size distribution models based on in situ measurements. J. Geophys. Res., 102 (D5), 6035-6045.

$\_,-$G. Ferry, and R. Pueschel, 1992; Aircraft studies of size- dependent aerosol sampling through inlets. J. Geophys. Res., 97, $3815-3824$.

Pruppacher, H. R, and J. D. Klett, 1998: Microphysics of Clouds and Precipitation. Kluwer Academic, $954 \mathrm{pp}$.

Reagan, J. A., 1995: New generation lidars to support aerosol radiation/climate forcing studies. Proc. IGARSS95, Florence, Italy, IEEE, 2313-2315.

, P. M. P. McCormick, and J. D. Spinhirne, 1989: Lidar sensing of aerosols and clouds in the troposphere and stratosphere. Proc. IEEE, 77 (3), 433-448.

Russell, P. B., T. J. Swissler, and M. P. McCormick, 1979: Methodology for error analysis and simulation of lidar aerosol measurements. Appl. Opt., 18, 3783-3797.

Sandford, M. C. W., 1967: Laser scatter measurements in the mesosphere and above. J. Atmos. Terr. Phys., 29, 1657-1662.

Shettle, E. P., and R. W. Fenn, 1979: Models for the aerosols of the lower atmosphere and the effects of humidity variations on their optical properties. AFGL-TR-79-0214, 94 pp. [Available from Air Force Geophysics Laboratory, Hanscom AFB, MA 01731.]

Spinhirne, J. D., J. A. Reagan, and B. M. Herman, 1980: Vertical distribution of aerosol extinction cross section and inference of aerosol imaginary index in the troposphere by lidar technique. J. Appl. Meteor., 19, 426-438.

Woodcock, A. H., 1953: Salt nuclei in marine air as a function of altitude and wind force. J. Meteor., 10, 362-371.

Young, S. A., D. R. Cutten, M. J. Lynch, and J. E. Davies, 1993: Lidar-derived variations in the backscatter-to-extinction ratio in southern hemisphere coastal maritime aerosols. Atmos. Environ., 27, 1541-1555.

Zhang, J., and H. Hu, 1997: Lidar calibration: A new method. Appl. Opt., 36, 1235-1238. 